\title{
Detecting Functional Connectivity of the Cerebellum Using Low Frequency Fluctuations (LFFs)
}

\author{
Yong He, Yufeng Zang, Tianzi Jiang, Meng Liang, and Gaolang Gong \\ National Laboratory of Pattern Recognition, Institute of Automation, Chinese Academy of \\ Sciences, 100080, Beijing, P. R. China \\ \{yhe, yfzang, jiangtz\}@nlpr.ia.ac.cn
}

\begin{abstract}
So far, resting state functional connectivity (RSFC) has been performed mainly by seed correlation analysis (SCA) on functional MRI (fMRI) studies. In previous studies, the seeds are usually selected on the basis of prior anatomical information or previously performed activation maps. In this paper, we proposed a novel way to select the desired seeds by taking the natures of resting state data into account. The proposed approach is based on the measurement of regional homogeneity ( $\mathrm{ReHo}$ ) of brain regions. Using this technique, 2 locations showing higher ReHo in the cerebellum (i.e. the bilateral anterior inferior cerebellum, AICb) were identified and used as the seeds for RSFC patterns studies. We found that the bilateral AICb show significant functional connectivity with the bilateral thalamus, the bilateral hippocampus, the precuneus, the temporal lobe and the prefrontal lobe. Further, the differences of RSFC patterns between the bilateral $\mathrm{AICb}$ were ascertained by a random effect paired $\mathrm{t}$ test. These findings may improve our understanding of cerebellar involvement in motor and a variety of non-motor functions.
\end{abstract}

\section{Introduction}

Functional connectivity in the context of functional brain imaging has been considered as a descriptive measure of spatiotemporal correlations between spatially distinct brain regions [1]. Functional connectivity in the human brain has traditionally focused on characterizing neuronal interactions between activated regions in a given experiment task [2]. Such task-specific functional connectivity (TSFC) may reflect coactivations resulting from the task itself and therefore strongly depend on the performance of the task. Recently, increasing attention has been focused on exploring functional dependencies between brain regions in no specific cognitive task, defined as resting state functional connectivity (RSFC). Some convincing evidence from functional MRI (fMRI) studies has demonstrated that there exists very low frequency fluctuations (LFFs) $(<0.08 \mathrm{~Hz})$ in MR signals measured in the resting brain [3-6]. In functionally related regions of the brain, these fluctuations are synchronous and exhibit high temporal coherence, even in those regions located remotely, which implies the existence of neuronal connectivity coordinating activity in the human brain. Thus, RSFC based on the LFFs may offer a more direct measure of functional interactions between brain regions since it is task-free. 
So far, RSFC has been studied in the motor cortex [3, 4], visual cortex [3, 4], language regions [5], and also in the subcortical regions, such as the thalamus [6] and the hippocampus $[6,7]$. Moreover, recent studies in patients have suggested that it would also be of specific clinical interest $[8,9]$.

Despite these studies, RSFC patterns in the cerebellum have not been reported in detail so far. As one of important subcortical structures in the brain, many investigations have indicated that the cerebellum not only contributes to the control of coordinated movement [10], but also may be involved in a variety of non-motor functions, including sensory discrimination [11], working memory [12] and emotional modulation [13]. It therefore would be very meaningful to ascertain which brain regions show significant functional connectivity with the cerebellum during rest. The investigation of RSFC patterns in the cerebellum may improve our understanding of cerebellar involvement in various functions.

One popular way for RSFC studies is seed correlation analysis (SCA), i.e. by selecting a predefined seed region of interest (ROI) as a reference and cross-correlating the time course of the seed with that of all other voxels in the whole brain to produce a functional network. In these studies, the seed ROIs are usually selected on the basis of prior anatomical information [6] or previously performed activation maps [3, 14]. However, such investigator-dependent selections may not be optimal for evaluating RSFC since the bias resulting from external effects (e.g. different experimental tasks) may make the connectivity patterns show completely different features. Intuitively, one should make full use of the information inferred from resting state data to select a relevant seed while evaluating RSFC using a SCA technique.

In this study, we propose a novel way to define the desired seed ROIs by taking into account the natures of resting state data. This approach is based on the measurement of regional homogeneity ( $\mathrm{ReHo}$ ) of brain areas. The ReHo may better represent the characteristics of brain areas in activity, as described by Zang et al. [15].

The paper is organized as follows: An experimental protocol in fMRI was first described. Then, the ReHo was reviewed and performed on resting state data; 2 locations showing higher ReHo in the cerebellum were identified and used as the seed leading to further RSFC analysis. Finally, a summary was provided.

\section{Methods}

\subsection{Data Acquisition}

FMRI studies of 34 healthy, right-handed volunteers (25 males; age range 22-40, mean 26.2) were performed with a 1.5-Tesla GE Signa scanner. Each subject was scanned for $400 \mathrm{sec}$ in a resting state. Functional images were acquired axially by using an EPI sequence with the following parameters: 2000/40 ms (TR/TE), 20 slices, $64 \times 64$ matrix, $90^{\circ} \mathrm{FA}, 24-\mathrm{cm}$ FOV, 5-mm thickness, and 1-mm gap.

\subsection{Data Preprocessing}

The first 10 time points of the resting state were discarded because of the instability of the initial MRI signal leaving 190 time points. The data were first preprocessed (slice 
timing, realignment, spatially normalization and re-sampled at $3 \mathrm{~mm}^{3}$ ) using SPM2 (www.fil.ion.ucl.ac.uk/spm). Then, in the AFNI package [17], linear drift was removed and the data were spatially smoothed using a 4-mm FWHM Gaussian kernel. After these, a low-pass frequency filter ( $<<0.08 \mathrm{~Hz}$ ) was applied to remove physiological high frequency noise [3]. The resulting LFFs were further analyzed.

\subsection{Regions of Interest (ROIs)}

In our previous study, the ReHo has been used to differentiate brain regions showing changes in activity between different states [15]. In this study, it was extended to define the seed ROIs located in the cerebellum. The description in detail is as follows:

Basic ReHo algorithm. In the context of the ReHo, it seems to be reasonable to assume that the voxels within a functional cluster have higher temporal homogeneity than that within a general cluster [15]. The ReHo of a cluster is measured using Kendall's coefficient of concordance $(K C C)$ [18]:

$$
W=\frac{\sum\left(R_{i}\right)^{2}-n(\bar{R})^{2}}{1 / 12 K^{2}\left(n^{3}-n\right)}
$$

where $W$ is the $K C C$ of a cluster, ranged from 0 to $1 ; R_{i}=\sum_{j=1}^{k} r_{i j}$, where $r_{i j}$ is the rank of the $i$-th time point in the $j$-th voxel; $\bar{R}=(n+1) k / 2 ; n$ is the number of time points of each voxel time series (here $n=190$ ); $k$ is the number of voxels in the cluster, (here $k=27$, for selecting the number of voxels, see ref [15]). Individual KCC maps were obtained on a voxel by voxel basis for each subject data set and further used to define the desired seed ROIs.

Definition of ROIs. The seed ROIs located in the cerebellum are defined as follows. First, a random effect one-sample t test [19] against 0.5 (i.e., $\mathrm{H}_{0}: K C C=0.5$, d.f. $=33$ ) was performed on the $K C C$ maps above. Voxels with $\mathrm{t}>15.52\left(P<1 \times 10^{-16}\right.$, uncorrected) and clusters volume $(\mathrm{CV})>540 \mathrm{~mm}^{3}$ were superimposed on high-resolution anatomical images (Fig.1). The resulting t-map shows higher ReHo in the bilateral anterior inferior cerebellum (AICb), the posterior cingulate cortex (PCC, Brodmann's area [BA] 23 and 31), etc. Here, we are just concerned about the bilateral AICb. Thus, anatomical location of peak voxel (i.e. highest $t$ value) within them was identified (Talairach coordinates: the left AICb [-26 -47 -32] and the right AICb [27 -47 -32]. Since the normalization of the data was performed in SPM2, we have transformed the coordinates of brain areas from the MNI space into the Talairach space using an algorithm proposed by http://www.mrc-cbu.cam.ac.uk/Imaging/Common/mnispace.shtml). Finally, each peak voxel obtained above and its nearest 26 neighbors were defined as a group ROI. Subject-specific ROIs were precisely consistent with the group ROIs anatomically. According to the above strategy, 2 subject-specific AICb ROIs for each subject were obtained leading to further RSFC analyses.

\subsection{Functional Connectivity Analyses}

RSFC patterns of the cerebellum were explored by a SCA approach on the basis of the seed ROIs above. Time series were first extracted for each subject-specific AICb 


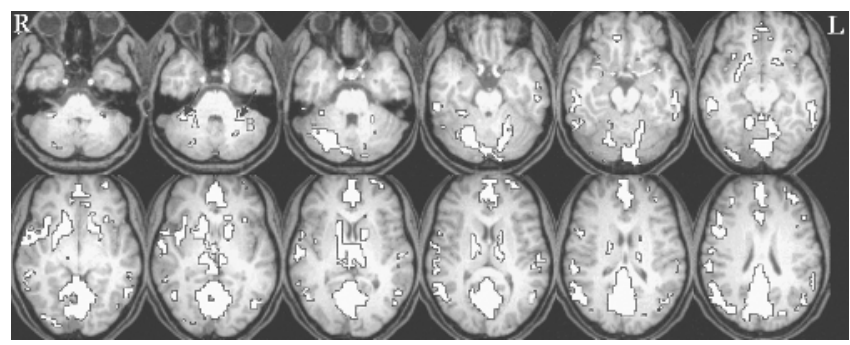

Fig. 1. A group t-map based on the individual KCC maps. The arrows indicate the locations of the bilateral AICb respectively. Z-axis from $Z=-36$ to $Z=+19$ in Talairach and Tournoux (TT) coordinates [16]. L indicates the left hemisphere of the brain. For other details, see Methods.

ROIs by averaging the time series of all the 27 voxels in the ROI. Then, the resulting time series was used as a reference for cross-correlation analysis (i.e. SCA). These correlation coefficients were further normalized using Fisher's $z$ transformation [20]:

$$
z=0.5 \log \left(\frac{1+r}{1-r}\right)
$$

where $r$ is the Pearson correlation coefficient at each voxel. The resulting $z$ values is approximately normal distribution with mean and

$$
\mu=0.5\left[\log \left(\frac{1+r_{\text {true }}}{1-r_{\text {true }}}\right)+\frac{r_{\text {true }}}{n-1}\right]
$$

standard deviation $\sigma \approx 1 / \sqrt{n-3}$. Finally, the individual $z$-maps entered into a random effect one-sample t-test [19] to determine brain regions showing significant functional connectivity across subjects. According to the above processing, 2 RSFC tmaps were obtained respectively. Voxels with $\mathrm{t}>9.917$ (d.f. $=33, P<1 \times 10^{-11}$, uncorrected) and $\mathrm{CV}>540 \mathrm{~mm}^{3}$ were superimposed on high-resolution anatomical images (Fig.2). The differences of RSFC patterns between the bilateral AICb was further ascertained by a random effect paired t-test performed on the $z$-maps above. Voxels with $|\mathrm{t}|>2.036$ (d.f. $=33, P<0.05$, uncorrected) and $\mathrm{CV}>540 \mathrm{~mm}^{3}$ were considered as significantly different between the RSFC patterns of the bilateral AICb.

The above procedures were coded in MATLAB (The MathWorks, Inc., Natick, MA) or processed in the AFNI package [17].

\section{Results}

Brain areas Showing Higher ReHo During Rest. Fig. 1 shows brain areas having higher ReHo during the rest, including the bilateral $\mathrm{AICb}$, the bilateral superior cerebellum (SCb), the PCC (BA 23 and 31) and the anterior cingulate cortex (ACC, BA 32). Here, we just focused on exploring the AICb as detailed in Methods.

RSFC Patterns of the Bilateral AICb. The right AICb showed significant RSFC with the following brain areas: the left $\mathrm{AICb}$, the bilateral $\mathrm{SCb}$, the bilateral thalamus (Th), the bilateral hippocampus (Hi), the left medial prefrontal cortex (MPFC, BA11), the 


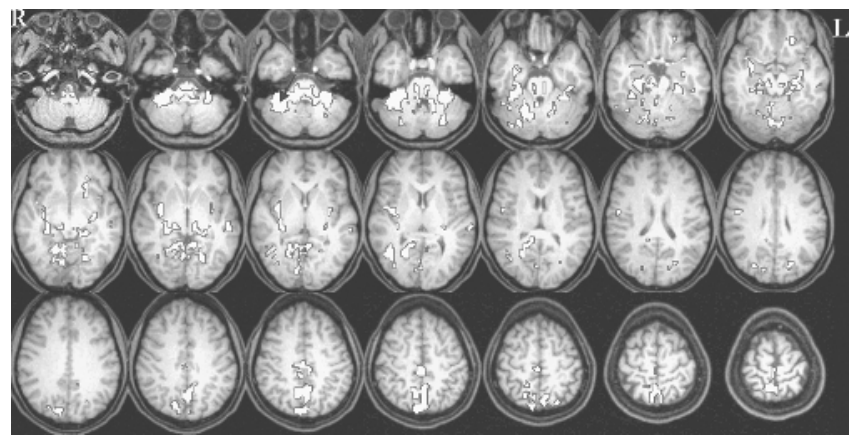

Fig. 2. RSFC t-map for the right AICb. The arrow indicates the location of the right AICb. Zaxis from $Z=-44$ to $Z=+60$ in TT coordinates. For other details, see Methods.

precuneus ( $\mathrm{PCu}, \mathrm{BA} 7)$ and the temporal lobe (BA21, 37 and 42) (Fig.2). The left $\mathrm{AICb}$ showed significant RSFC with the following brain regions: the right $\mathrm{AICb}$, the bilateral SCb, the bilateral Th, the bilateral Hi, the PCC (BA 23), the PCu (BA 7) and the supplement motor area (SMA, BA 6) (The figure is not given here).

The Differences of RSFC Patterns Between the Bilateral AICb. Fig. 3 shows the differences of RSFC patterns for the bilateral AICb detected by a paired t-test. Of 29 brain regions with significant differences, 14 showed higher connectivity with the right $\mathrm{AICb}$, compared with the left $\mathrm{AICb}$. Further details regarding these brain regions were presented in Table 1.

\section{Discussion}

In this study, we used a novel way, the ReHo, to define the desired seed ROIs while exploring RSFC patterns of brain regions using a SCA method. As compared to other methods used for the selection of the seed in the resting state, e.g., anatomically knowledge-based or task activation-based methods, the main advantage of ReHo is that it makes full use of the nature of the resting state data. Using this technique, we identified the seed ROIs located in the bilateral $\mathrm{AICb}$ and ascertained their connectivity patterns during rest. Results demonstrate that the bilateral AICb show significant functional connectivity with a distinct set of brain regions during rest, thus providing insight into understanding functional pathways related to the cerebellum.

A large body of evidences has shown that the cerebellum not only participates in motor control, but also may be involved in a variety of non-motor functions. The explorations about functional pathways related to the cerebellum would be very significant for understanding of how the cerebellum works. However, these studies so far have usually focused on exploring TSFC patterns related to the cerebellum [23, 26]. Obviously, such patterns strongly depend on the performance of the tasks. In contrast, RSFC analysis based on the LFFs may provide a more direct measure for neural circuitry. To probe the possibility, we mapped the connectivity patterns for the bilateral $\mathrm{AICb}$ during rest respectively. Results show that the right $\mathrm{AICb}$ has significant functional connectivity with the left $\mathrm{AICb}$, the bilateral $\mathrm{Th}$, the bilateral $\mathrm{Hi}$, the 


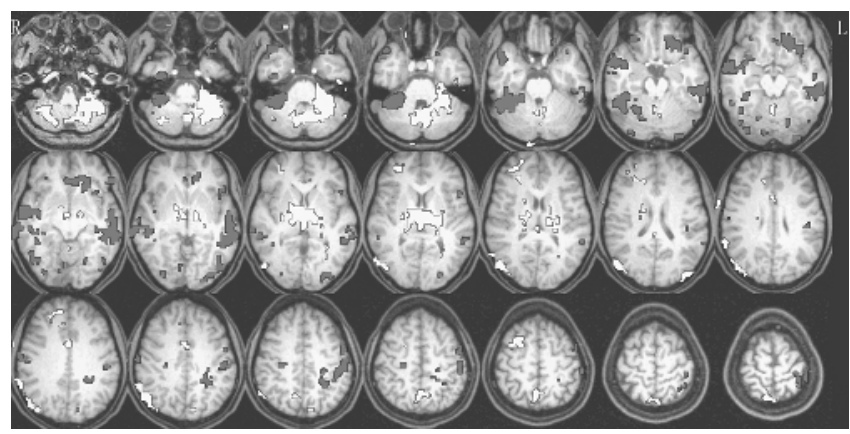

Fig. 3. Paired t-test for the differences of RSFC patterns between the bilateral AICb. Z-axis from $\mathrm{Z}=-44$ to $\mathrm{Z}=+60$ in TT coordinates. White cluster: the left $\mathrm{AICb}>$ the right $\mathrm{AICb}$. Grey cluster: the right $\mathrm{AICb}>$ the left AICb. For other details, see Methods and Table 1.

Table 1. Paired t-test for the difference of RSFC patterns between the bilateral AICb.

\begin{tabular}{|c|c|c|c|c|c|c|c|c|c|c|c|}
\hline \multicolumn{6}{|c|}{ Left $<$ Right } & \multicolumn{6}{|c|}{ Left $>$ Right } \\
\hline $\mathrm{k}$ & $\mathrm{x}$ & $\mathrm{y}$ & $\mathrm{z}$ & $\mathrm{t}$ & Regions, BA & $\mathrm{k}$ & $\mathrm{x}$ & $\mathrm{y}$ & $\mathrm{z}$ & $\mathrm{t}$ & Regions, BA \\
\hline 1306 & 27 & -47 & -32 & -26.8 & R.AICb & 1227 & -24 & -49 & -32 & 27.4 & L.AICb \\
\hline 352 & -21 & 28 & -9 & -4.39 & L.MPFC, 10 & 46 & -40 & -92 & 17 & 5.20 & L.OL, 19 \\
\hline 601 & -55 & -32 & -13 & -4.19 & L.MTG, 20 & 711 & 9 & -23 & 8 & 4.26 & R.Th \\
\hline 23 & 25 & 31 & -10 & -3.67 & R.MPFC, 11 & & -11 & -14 & 6 & 4.09 & L.Th \\
\hline 108 & -24 & -92 & -1 & -3.65 & L.OL, 18 & 418 & 58 & -48 & 24 & 4.06 & R.IPC, 40 \\
\hline 300 & -21 & -44 & 30 & -3.64 & L.WM & 118 & 21 & -72 & -42 & 3.91 & R.PICb \\
\hline 32 & 26 & -35 & 39 & -3.44 & R.WM & 28 & 22 & 35 & 22 & 3.38 & R.SFG, 10 \\
\hline 102 & -42 & -33 & 41 & -3.40 & L.M1, 4 & 62 & 1 & 2 & 35 & 3.28 & R.CG, 24 \\
\hline 48 & 58 & -15 & 34 & -3.19 & R.PrCG, 6 & 176 & 2 & -60 & 51 & 2.93 & R.PCu, 7 \\
\hline 48 & -27 & -92 & -21 & -3.14 & L.SCb & 57 & 19 & 0 & 55 & 2.91 & R.MFG, 6 \\
\hline 48 & 26 & -90 & -2 & -3.04 & R.OL, 18 & 27 & 10 & 46 & 35 & 2.86 & R.SFG, 9 \\
\hline 31 & 7 & -78 & -5 & -2.94 & R.PCC, 18 & 26 & -8 & -84 & 33 & 2.85 & L.Cu, 19 \\
\hline 55 & -45 & -6 & 45 & -2.91 & L.PrCG, 6 & 89 & 27 & 48 & 12 & 2.82 & R.MFG, 10 \\
\hline \multirow[t]{2}{*}{36} & -45 & 13 & 10 & -2.70 & L.IFG, 44 & 29 & -26 & -59 & 6 & 2.70 & L.WM \\
\hline & & & & & & 27 & 16 & 13 & 31 & 2.58 & R.ACC, 32 \\
\hline
\end{tabular}

Left part: results showing more significant RSFC with the right AICb. Right part: results showing more significant RSFC with the left AICb. k, cluster size in voxels; BA, Brodmann's area; $\mathrm{x}, \mathrm{y}, \mathrm{z}$, coordinates of TT. Abbreviations: $\mathrm{AICb}$, anterior inferior cerebellum; MPFC, medial prefrontal cortex; MTG, middle temporal gyrus; MFG, middle frontal gyrus; OL, occipital lobe; M1, primary motor cortex; PrCG, precentral gyrus; SCb, superior cerebellum; PCC, postulate cingulate cortex; IFG, inferior frontal gyrus; Th, thalamus; IPC, inferior parietal cortex; PICb, posterior inferior cerebellum; WM, white matter; SFG, superior frontal gyrus; CG, cingulate gyrus; $\mathrm{PCu}$, Precuneus; ACC, anterior cingulate cortex.

PCu, the left MPFC and the temporal lobe (Fig. 2). Moreover, RSFC pattern of the left $\mathrm{AICb}$ was very similar to that of the right $\mathrm{AICb}$. The findings were strongly sup- 
ported by quite a few previous studies. First, of the regions above, the bilateral Th and the Hi have been shown significant RSFC with the cerebellum while they are selected as the seeds respectively leading to a SCA [6]. Second, Middleton and Strick's study of using transneuronal tracing methods in monkeys has indicated there are ipsi- and contra-lateral connections between cerebellum and prefrontal cortex related by the mediodorsal (and other) thalamic nuclei [24]. Third, in the "cognitive dysmetria" model, the cerebellum has been considered as a key structure in a cerebellar-thalamicprefrontal circuit [25]. Our findings may provide a new insight to understand the cerebellar involvement in motor control and cognitive functions.

Despite the similarity for the bilateral AICb RSFC maps and the similar symmetry in anatomical locations, paired t-test still demonstrates that there exist significant differences in a widely variety of brain regions (Fig. 3, Table 1). Of 29 brain regions with significant differences, 14 showed higher connectivity with the right $\mathrm{AICb}$, compared with the left AICb. Interestingly, we found that the left M1 shows more significant functional connectivity with the right $\mathrm{AICb}$ than that with the left $\mathrm{AICb}$, however, we did not observe similar phenomenon for the right M1. In addition, the temporal lobe and the prefrontal lobe were observed significant connectivity with the right $\mathrm{AICb}$, but the bilateral Th and the right SFG show more with the left AICb.

Although we have mapped connectivity patterns in the AICb during rest, brain's functional connectivity is highly flexible and can be modulated for a given changing environment. Our recent study has indicated that functional connectivity of the cerebellum varies from the resting state to the motor task [21]. The modulation is very important for the brain to execute different function in different brain states. In addition, recent evidence from TSFC studies has indicated that altered functional pathways related to the cerebellum appear in the schizophrenia $[23,26]$. As an alternative to TSFC analysis, our methods may show more powerful ability in clinical studies, especially for those patients with progressed state of the disease since it is task-free.

It is important to emphasize that, in this study, the connectivity patterns of the $\mathrm{AICb}$ during rest were explored by combining the SCA and the ReHo. Besides the $\mathrm{AICb}$, our finding that the PCC may be very active (higher ReHo) is consistent with a recent study that the PCC is considered as a critical node during rest [14]. It may provide a powerful support for the validity of our methods. Anyway, the selection of the seed based on the ReHo seems to be more reasonable, compared with conventional methods. The present study could also be generalized to ascertain RSFC patterns of other brain regions, and especially to explore altered functional connectivity between the patients and the controls.

Acknowledgment. This work was partially supported by the Hundred Talents Programs of the Chinese Academy of Sciences, the Natural Science Foundation of China, Grant No. 60172056 and 60121302.

\section{References}

1. Friston KJ, Frith CD, Liddle PF, Frackowiak RS: Functional connectivity: the principal component analysis of large (PET) data sets. J Cereb Blood Flow Metab 13 (1993) 5-14.

2. Homae F, Yahata N, Sakai KL: Selective enhancement of functional connectivity in the left prefrontal cortex during sentence processing. NeuroImage 20 (2003) 578-586. 
3. Biswal B, Yetkin FZ, Haughton VM, Hyde JS: Functional connectivity in the motor cortex of resting human brain using echo-planar MRI. Magn Reson Med 34 (1995) 537-541.

4. Lowe MJ, Mock BJ, Sorenson JA: Functional connectivity in single and multislice echoplanar imaging using resting state fluctuations. Neuroimage 7 (1998) 119-132.

5. Hampson M, Peterson BS, Skudlarski P, Gatenby JC, Gore JC: Detection of functional connectivity using temporal correlations in MR images.Hum Brain Mapp 15(2002)247262.

6. Stein T, Moritz C, Quigley M, et al. Functional connectivity in the thalamus and hippocampus studied with fMRI. Am J Neuroradiol 21 (2000) 1397-1401.

7. Rombouts SA, Stam CJ, Kuijer JP, Scheltens P, Barkhof F: Identifying confounds to increase specificity during a "no task condition". Evidence for hippocampal connectivity using fMRI. Neuroimage 20 (2003) 1236-1245.

8. Lowe MJ, Phillips MD, Lurito JT, Mattson D, Dzemidzic M, Mathews VP: Multiple sclerosis: low-frequency temporal blood oxygen level-dependent fluctuations indicate reduced functional connectivity_-initial results. Radiology 224 (2002) 184-192.

9. Li SJ, Biswal B, Li Z, Risinger R, Rainey C, Cho JK, Salmeron BJ, Stein EA: Cocaine administration decreases functional connectivity in human primary visual and motor cortex as detected by functional MRI. Magn Reson Med 43 (2000) 45-51.

10. Thach WT, Goodkin HP, Keating JG: The cerebellum and the adaptive coordination of movement. Annu Rev Neurosci. 15 (1992) 403-442.

11. Gao JH, Parsons LM, Bower JM, Xiong J, Li J, Fox PT: Cerebellum implicated in sensory acquisition and discrimination rather than motor control. Science 272 (1996) 545-547.

12. Klingberg T, Kawashima R, Roland PE: Activation of multi-modal cortical areas underlies short-term memory. Eur J Neurosci 8 (1996) 1965-1971.

13. George MS, Ketter TA, Parekh PI, Horwitz B, Herscovitch P, Post RM: Brain activity during transient sadness and happy in healthy women. Am J Psychiatry 152(1995)341351.

14. Greicius MD, Krasnow B, Reiss AL, Menon V: Functional connectivity in the resting brain: A network analysis of the default mode hypothesis, Proc Natl Acad Sci 100(2003) 253-258.

15. Zang YF, Jiang TZ, Lu YL, He Y, Tian LX: Regional homogeneity approach to fMRI data analysis. NeuroImage 22(2004) 394-400.

16. Talairach J, Tournoux PA: Coplanar stereotactic atlas of the human brain, Thieme, (1988).

17. Cox, RW: AFNI software for analysis and visualization of functional magnetic resonance neuroimages. Comput Biomed Res 29 (1996) 162-173.

18. Kendall M, Gibbons JDR: Correlation methods. Oxford: Oxford University Press (1990).

19. Holmes AP, Friston KJ: Generalisability, random effects \& population inference. NeuroImage 7 (1998) 754.

20. Press WH, Teukolsky SA, Vetterling WT, Flannery BP (1992): Numerical recipes in C, 2 ed. U.K. Cambridge Univ. Press.

21. Jiang TZ, He Y, Zang YF, Weng XC: Modulation of functional connectivity during the resting state and the motor task. Hum Brain Mapp 22 (2004) 63-71.

22. Schmahmann JD: From movement to thought: anatomic substrates of the cerebellar contribution to cognitive processing. Hum Brain Mapp 4 (1996) 174-198.

23. Stephan KE, et al. Effects of olanzapine on cerebellar functional connectivity in schizophrenia measured by fMRI during a simple motor task. Psychol Med.31 (2001) 1065-1078.

24. Middleton FA, Strick PL: Anatomical evidence for cerebellar and basal ganglia involvement in higher cognitive function. Science 266 (1994) 458-461. 
25. Andreasen NC, O'leary DS, Cizadlo T, Arndt S, Rezai K, Ponto LL, Watkins GL, Hichwa RD: Schizophrenia and cognitive dysmetria: A positron-emission tomography study of dysfunctional prefrontal-thalamic-cerebellar circuitry. Proc Natl Acad Sci 93(1996) 99859990.

26. Schlosser R, Gesierich T, Kaufmann B, Vucurevic G, Hunsche S, Gawehn J, Stoeter P: Altered effective connectivity during working memory performance in schizophrenia: a study with fMRI and structural equation modeling. Neuroimage 19 (2003) 751-763. 